\title{
William T. O'Brien Sr. (Ed.), Top 3 Differentials in Neuroradiology: A Case Review. Thieme Medical Publishers, Inc., 2015. ISBN 978-1- 60406-723-1
}

\author{
Davide D'Arienzo $^{1} \cdot$ Luigi Mansi $^{1}$
}

Published online: 19 March 2016

(C) Springer-Verlag Berlin Heidelberg 2016

This original and voluminous publication has been edited by William T. O'Brien, Program Director of the Diagnostic Radiology Residency at the David Grant USAF Medical Center of the Travis Air Force Base, California, and Associate Clinical Professor of Radiology at the University of California, Sacramento.

The 720-page book, including 732 high-quality images, describes and discusses 300 clinical cases, which are organized into three sections corresponding to the three neuroradiology subspecialties: brain, head and neck, and spine.

The exposition of each case begins with the presentation of the images and a brief description of the patient's clinical features, followed by key imaging findings. The author then provides the "top 3" differential diagnoses and the final diagnosis, and closes with some clinical pearls and reflections on the described case and the associated pathological condition.

The first section, the Brain, is subdivided into four subsections: congenital and developmental, attenuation and signal abnormality, masses and mass-like lesions, and vasculature and cerebrospinal fluid spaces. The second section, Head and Neck, is divided into six subsections: calvarium and skull base, temporal bone, sinonasal, maxillofacial, neck, and orbits. The third section, Spine, is a single unit, with no subdivision of the corresponding cases into separate sections.

At the end of the book, the author provides an index of differential diagnoses by case and an index of the key findings (ordered by case number within each section).

Thanks to the well-organized didactic structure that involves the reader as protagonist in achieving the final decision, this text can serve as an easy-to-use practical manual and virtually comprehensive tool not only for neuroradiologists, but for clinicians and surgeons who deal with neuroradiological imaging as well.

The book can be particularly helpful for residents and students, as its editorial perspective guides the reader through a critical evaluation of all data present and/or associated with a diagnostic image. In this sense, the relevance of clinical and epidemiological information is emphasized as the primary component, leading to a final response based on the knowledge of all elements involved in the diagnostic reading of a "radiographic plate".
Luigi Mansi

luigi.mansi@unina2.it

1 Second University of Naples, Naples, Italy 